\title{
The role of eosinophils in asthma
}

\author{
Faris Q. Alenzi ${ }^{{ }^{\star}}$, Fahad G. B. Alanazi ${ }^{2}$, Abdulaziz D. Al-Faim ${ }^{3}$, Mohamed W. Al-Rabea ${ }^{4}$, \\ Waleed Tamimi ${ }^{5}$, Bassel Tarakji ${ }^{6}$, Omar Kujan ${ }^{6}$, Ali Al-Jabri ${ }^{7}$, Richard K. H. Wyse ${ }^{8}$ \\ ${ }^{1}$ College of Applied Medical Sciences, Salman bin Abdulaziz University, Al-Kharj, Saudi Arabia; \\ *Corresponding Author: falenzi@ksu.edu.sa \\ ${ }^{2}$ Department of Pharmacy, Ministry of Health, Riyadh, Saudi Arabia \\ ${ }^{3}$ Department of Preventive Medicine, Ministry of Health, Riyadh, Saudi Arabia \\ ${ }^{4}$ College of Medicine, King Abdulaziz University, Jeddah, Saudi Arabia \\ ${ }^{5}$ Department of Pathology and Laboratory Medicine, King Abdulaziz Medical City, Riyadh, Saudi Arabia \\ ${ }^{6}$ Department of Pathology, Al-Farabi Medical College, Riyadh, Saudi Arabia \\ ${ }^{7}$ Department of Microbiol \& Immunology, College of Medicine, Sultan Qaboos University, Muscat, Oman \\ ${ }^{8}$ Imperial College of Medicine, London, UK
}

Received 17 December 2012; revised 15 January 2013; accepted 22 January 2013

\section{ABSTRACT}

Asthma is a chronic inflammatory disorder of the airways characterized by recurring episodes of reversible airway obstruction, hyper-responsiveness, wheezing, breathlessness and coughing. Clinical diagnosis of asthma is based on the pattern of clinical symptoms and pulmonary fuction tests. Asthma affectes $5 \%-10 \%$ of the population and the number of worldwide cases is approximately $\mathbf{3 0 0}$ milliones. The incidence of this disease is increasing particulry in western countries [1]. It is the cause of a huge economic burden to national healthcare services. In a minority of cases, asthma is potentially fatal. After a period when fatalities appeared to be increasing [2], in recent years asthma-related mortality has progressively declined due to the development of specific asthma disease management programs, as well as the extensive use of inhaled corticosteroids [3]. Inflammation of the airways is a central component in asthma. Inflammation is associated with infliltration of the airway wall with eosinophiles and or neutronphiles mast cell degranulation and $T$ cell activetion. Other pathological features include, subbasement membrane thickening, loss of epithetlial cell integrity, goblet cells hyperplasia Increase in airway smooth muscle mass. Eosinophils are thought to be vital in the development of airway hyperreactivity, with the eosinophil cationic protein playing a crucial role [4]. The fact that treatment of asthma with corticosteroids reduces eosinophils numbers and decreases airway reactivity further supports this hypothesis.
Keywords: Eosinophils; Asthma

\section{ROLE OF EOSINOPHILS IN ASTHMA}

Eosinophils are formed in bone marrow and are terminally differentiated cells. In disease-free inviduals most eosinophils are primarily located in the gut, and can survive for weeks, whilst their half-life in the circulation is less than a day. The presence of eosinophils in significant numbers in skin, or in the airways, is found in a variety of disease processes [5-7]. Eosinophils are innate immune cells possessing specific cytoplasmic granules that contain cationic toxins capable of disabling many pathways. Eosinophils are derived from $\mathrm{CD}_{3}{ }^{+}$pluripotent progenitor cells, a process utilizing Th2 cytokines IL-3 and granulocye-monocyte colony stimulating factor (GM-CSF) as early precursors. IL-5 involves with priming the cells and in the terminal differentiation of eosinophils [8]. IL-5 also acts in acute disease response by releasing large numbers of eosinophils from the bone marrow into the systemic circulation.

Bronchial eosinophilia occurs in both atopic (extrinsic) and non-atopic (intrinsic) asthma. There is a clear relationship between asthma exacerbation rates and sputum eosinophil concentrations. Eosinophil influx into the lungs from capillaries is mediated by by vascular cell adhesion molecule-1 (VCAM-1), eotaxin, granulocytemacrophage colony-stimulating factor (GM-CSF), interleukin-4 (IL-4) and IL-5. During an asthma attack, eosinophils are stimulated to release proinflammatory and cytotoxic mediators, and growth factors, causing augmantation of the inflammatory process. These events might be responsible for bronchial hyperresponsiveness and smooth muscle contraction, vascular leakage, hypersecretion of mucus, and shedding of epithelial cells. Eosinophils may also initiate the process of tissue repair 
through the release of cytokines and growth factors. IL-4 increases levels of vascular cell adhesion molecule-1 (VCAM-1) in endothelial and airway epithelial cells, and may represent a crucial factor in eosinophil trafficking [9].

\section{ROLE OF IL-5 IN ALLERGIC EOSINOPHILIC DISEASE}

IL-5 is necessary for the production, maturation, accumulation, and activation of eosinophils, as well as their survival [10]. It is also involved in eosinophil chemoattraction. IL-5 appears to represent an ideal drug development target to ameliorate eosinophil toxicity in the asthmatic lung since it has good efficacy and is selective. In an in vivo models of asthma, it has been shown that inhibiting the effects of IL-5 using specific monoclonal antibodies decreases both blood and bronchoalveolar eosinophilia and modulate the response of the lung to antigene challenge.

The Inhibition of IL-5 can prevent tissue damage due to eosinophil accumulation and airway inflammation [11]. In addition, treatment of wild type mice with neutralizing anti-IL-5 mAb preventes sub-epithelial and peribronchial fibrosis; this suggests that eosinophils are involved in allergen-induced sub-epithelial and peribronchial fibrosis, which could be possibly mediated via TGF- $\beta 1$ [12]. Several trials treating asthmatic patients with humanized antibodies against IL-5 have not shown positive clinical outcomes. The randomised, doubleblinded, placebo-controlled, parallel-group clinical trial by Leckie et al. [13] which was designed to validate the safety and activity of the humanised anti-IL-5 mAb Mepolizumab (SB240563), while showing significant reductions both in blood eosinophils and sputum eosinophils for at least 1 month, offered no significant effect on pulmonary functions following allergen challenge [14]. Technical aspects of this clinical trial were subsequently criticized [15].

A clinical trial of a humanised anti-IL-5 mAb Mepolizumab in mild asthma patients was lator reported by Flood-Page [16]. Whilst the antibody was shown to have the potential to slow the increases in airway and bone marrow eosinophils, it gave no clinical benefit to the asthmatic patients in the study. Using the humanised anti-IL-5 mAb Reslizumab (SCH55700) similar results were reported in patients with severe asthma chosen for their lack of symptomatic control when using inhaled corticosteroid use.

More recently, two clinical trials showed clinical benefits when asthmatics were phenotyped [17]. An alternative approach has been molecular modelling of the IL-5 receptor $\alpha$-chain to construct specific receptor antagonists. The compound, YM-90709, was shown to be a selective inhibitor of the IL-5R [18]. Despite the use of humanised anti-IL-5 mAb initially providing disappointing clinical outcomes, more recently treatment of severe asthmatics with high eosinophils using an anti-IL-5 $\mathrm{mAb}$ has been found to reduce the number of exacerbations [19], and was suggested to be a potential effective approach for the long-term treatment of patients with eosinophilic disorders including asthma [20].

Eosinophils may be also important in the pathophysiology of airway remodelling. TGF $\beta$ release, thickening of sub-epithelial basement membranes are associated with bronchial mucosal eosinophils particularly in severe asthmatics [21].

Treatment of asthma patients with Mepolizumab, to reduce airway eosinophil numbers, considerably reduces the expression of tenascin (an extracellular matrix glycoprotein involved with wound healing), lumican (which binds collagen fibrils and regulates interfibrillar spacings), and procollagen III (a fibrillar collagen present in extensible connective tissues such as lung) in the bronchial mucosal reticular basement membrane, whilst antiIL-5 treatment reduces numbers of airway eosinophils expressing mRNA for TGF- $\beta 1$ and the concentration of TGF- $\beta 1$ in bronchoalveolar lavage fluid [16]. It was concluded that eosinophils might contribute to tissue remodelling processes in asthma by regulating the extracellular matrix protein deposition and anti-IL-5 mAb may prove useful in preventing this.

\section{ROLE OF AIRWAY EPITHELIUM IN ENGULFING APOPTOTIC EOSINOPHILS}

The airway epithelium is predominantly comprised of ciliated, nonciliated, and basal cells. Epithelial cell damage resulting in cilial dysfunction and loss represents a core characteristic of asthma pathogenesis and is considered an important contributor to hyperresponsiveness of the airways.

Walsh's group has already shown [22] that an apoptotic differentiated eosinophilic cell line (EoL-1) was distinguished via specific recognition mechanisms, and then ingested, by the large bronchial airway epithelial human cells, LAEC and A549, and that this process is up-regulated by the pro-inflammatory cytokine IL- $1 \beta$ and the glucocorticoid dexamethasone. Treatment of EoL-1 cells with dexamethasone generates a large increase in apoptosis, suggesting that the differentiated EoL-1 apoptotic response to glucocorticoids is similar to primary human eosinophils [23,24]. SAEC, LAEC and A549 have a similar capacity and receptor-usage when recognising and engulfing apoptotic eosinophils, although neither resting nor cytokine stimulation SAEC, LAEC, or A549 recognise or ingest apoptotic neutrophils [25]. Treating asthma with glucocorticoid therapy reduces airway inflammation by inhibiting cytokine pro- 
duction and by stimulating PBE clearance [26]. High concentrations of glucocorticoids are known to induce eosinophil apoptosis in vitro [27,28]. Chauhan et al. [29] have shown that, when using low serum-containing media, treatment of undifferentiated EoL-1 cells with dexamethasone induced an apoptotic pathway that was inhibited by IL-5. Using confocal microscopy, our own results demonstrated that EoL-1 cells are localized to the surface of A549 and are enclosed by a dense concentration of F-actin, an observation suggestive of phagocytic cup formation by the phagocyte. That stimulation of LAEC or A549 with IL- $1 \beta$ enhances the phagocytic capacity for apoptotic differentiated EoL-1 may be related to the fact that the same cytokine is believed to promote eosinophil accumulation via augmenting eotaxin production by the airway epithelium [30].

It seems likely that cytokines concerned in the accumulation of eosinophils would also prepare resident cells to remove apoptotic eosinophils, in an analogous manner to the clearance of apoptotic neutrophils by cytokinestimulated monocyte-derived macrophages [31].

The benefical action of glucocorticoids to reduce eosinophilic inflammation may entail an increase of eosinophil apoptosis $[28,32]$ and ensuing detection and engulfment by alveolar macrophages [33]. Treatment of LAEC and A549 with glucocorticoids enhances the numbers of cells engulfing apoptotic differentiated EoL-1. In concordance with this is that Liu et al. [34] has established the potentiation of nonphlogistic clearance of apoptotic neutrophils and eosinophils by inflammatory macrophages following exposure to glucocorticoids, but does not occur with non-glucocorticoid steroids.

How apoptotic cells are recognised appears to relate to the specific cell type responsible for their engulfment. That lectin and integrin are involved in the recognition of apoptotic cells has been described in several studies:

- An uncharacterised lectin-dependent interaction [35];

- CD36 and $\alpha \mathrm{v} \beta 3$ involvement in identification by macrophages of apoptotic eosinophils/neutrophils [36];

- $\alpha \mathrm{v} \beta 5$ has been causally linked to the recognition of apoptotic cells by immature dendritic cells [37]; and rod outer segments by retinal pigmented epithelium [38];

- A stereo-specific recognition of phosphatidylserine expressed on apoptotic cells after loss of membrane asymmetry $[39,40]$;

- Macrophage scavenger receptors [41,42].

Additionally, Walsh's laboratory has shown [43] that the amino sugars glucoseamine, n-acetyl glucosamine, and galactosamine significantly inhibit uptake of aged human apoptotic differentiated EoL-1 by LAEC or A549. The tetrapeptide Arg-Gly-Asp-Ser (RGDS) has been used to blockade integrin interactions between differenttiated EoL-1 and airway epithelium since it contains the ubiquitously recognised RGD motif for integrins. Furthermore, incubation of apoptotic differentiated EoL-1 with RGDS significantly reduced their uptake by LAEC or A549, whilst a control tetrapeptide (RGES) (define acronym) showed no effect on ingestion of apoptotic differentiated EoL-1. Monoclonal antibodies to $\alpha \mathrm{v} \beta 3$, $\alpha \mathrm{v} \beta 5$, $\beta 5$, and CD36 inhibit the phagocytic process which underlines their individual roles in the processes involved. These findings are consistent with previous work using apoptotic human peripheral blood eosinophils (PBE) $[24,44]$. Furthermore, flow cytometry has demonstrated that $\alpha \mathrm{v} \beta 3, \alpha \mathrm{v} \beta 5$ and CD36 are expressed at midhigh levels in both LAEC and A549, while the subunit $\beta 5$ was expressed in A549 epithelial cells.

The exposure of phosphatidylserine in the outer leaflet of the plasma membrane represents a conspicuous change on the surface of apoptotic cells. We demonstrated inhibition of uptake of apoptotic differentiated EoL-1 after mAb-dependent blockade of the putative phosphatidylserine receptor (PSR). We have extended previous findings for PSR in phagocyte-apoptotic cell interactions [45-50] to demonstrate a role for this recaptor in epithelial cell detection of apoptotic differentiated EoL-1, and this receptor may well be responsible for inducing the release of the important antinflammatory, antiimmunogenic molecule, TGF- $\beta$.

\section{CONCLUSION}

Selective eosinophil accumulation in lung tissue, characteristic of asthmatic inflammation, is concomitant with activation and release of apotent pro-inflammatory cocktail of granule-derived basic proteins, mediators, cytokines and chemokines. A number of approaches, as outlined in this review, seek biochemically to diminish the impact of asthmatic inflammation, and encouraging clinical results are starting to emerge, most particularly when using anti-IL-5 mAb. The use of glucocorticoids and the amino sugars glucoseamine, n-acetyl glucosemine, and galactosamine, also appear promising approaches and may eventually play an important future role in curbing tissue damage due to eosinophil accumulation during airway inflammation.

\section{REFERENCES}

[1] (2008) World Health Organisation. http://www.who.int/mediacentre/factsheets/fs307/en/print .html

[2] Sears, M.R., Barnes, P.J., Rodger, I.W. and Thomson, N.C. (1998) Epidemiology. Asthma: Basic mechanisms and clinical management. Academic Press, San Diego, 1-33.

[3] Rubin, B.K. and Pohanka, V. (2012) Beyond the guidelines: Fatal and near-fatal asthma. Paediatric Respiratory Reviews, 13, 106-111. doi:10.1016/j.prrv.2011.05.003 
[4] Bystrom, J. and Amin, K. (2011) Bishop-bailey D. Analysing the eosinophil cationic protein-A clue to the function of the eosinophil granulocyte. Respiratory Research, 12, 10. doi:10.1186/1465-9921-12-10

[5] Venge, P.E. (1998) Asthma: Basic mechanisms and clinical management. In: Barnes, P.J., Rodger, I.W. and Thomson, N.C., Eds., Academic Press, San Diego, 1-33.

[6] Gleich, G.J., Adolphson, C.R. and Leiferman, K.M. (1993) The biology of the eosinophilic leukocyte. Annual Review of Medicine, 44, 85-101.

doi:10.1146/annurev.me.44.020193.000505

[7] Spry, C.J.F. (1998) Eosinophils: A comprehensive review and guide to the scientific and medical literature. Oxford University Press, Oxford, 1998.

[8] Denburg, J.A. (1998) The origins of basophils and eosinophils in allergic inflammation. Journal of Allergy and Clinical Immunology, 102, S74-S76. doi:10.1016/S0091-6749(98)70034-X

[9] Rosenberg, H.F., Phipps, S. and Foster, P.S. (2007) Eosinophil trafficking in allergy and asthma. Journal of Allergy and Clinical Immunology, 119, 1303-1310. doi:10.1016/j.jaci.2007.03.048

[10] Sanderson, C.J. and Urwin, D. (2000) Interleukin-5: A drug target for allergic diseases. Current Opinion in Investigational Drugs, 1, 435-441.

[11] Greenfeder, S., Umland, S.P., Cuss, F.M., Chapman, R.W. and Egan, RW. (2001) Th2 cytokines and asthma. The role of interleukin-5 in allergic eosinophilic disease. Respiratory Research, 2, 71-79. doi:10.1186/rr41

[12] Tanaka, H., Komai, M., Nagao, K., Ishizaki, M., Kajiwara, D., Takatsu, K., Delespesse, G. and Nagai, H. (2004) Role of interleukin-5 and eosinophils in allergen-induced airway remodeling in mice. American Journal of Respiratory Cell and Molecular Biology, 31, 62-68. doi:10.1165/rcmb.2003-0305OC

[13] Leckie, M.J., tenBrinke, A., Khan, J., Diamant, Z., O’Connor, B.J., Walls, C.M., et al. (2000) Effect of interleukin-5 blocking monoclonal antibody on eosinophils, airway hyperresponsiveness and the late asthmatic response. Lancet, 356, 2144-2148. doi:10.1016/S0140-6736(00)03496-6

[14] O’Byrne, P.M., Inman, M.D. and Parameswaran, K. (2001) The trials and tribulations of IL-5, eosinophils, and allergic asthma. Journal of Allergy and Clinical Immunology, 108, 503-508. doi:10.1067/mai.2001.119149

[15] Lipworth, B.J. (2001) Eosinophils and airway hyperresponsiveness. Lancet, 357, 1446. doi:10.1016/S0140-6736(00)04597-9

[16] Flood-Page, P.T., Menzies-Gow, A.N., Kay, A.B. and Robinson, D.S. (2003) Eosinophil's role remains uncertain as anti-interleukin-5 only partially depletes numbers in asthmatic airway. American Journal of Respiratory and Critical Care Medicine, 167, 199-204. doi:10.1164/rccm.200208-7890C

[17] Huib, A.M.K., Michael, E., Ronald, D., Pierluigi, P., Ekkehard, B., Mark, V., Ralf, S., Dipl, M., Wolfgang, S., Petra, M.-Z. and Eric, D.B. (2012) Tiotropium in asthma poorly controlled with standard combination therapy. The
New England Journal of Medicine, 367, 1198-1120

[18] Morokata, T., Ida, K. and Yamada, T. (2002) Characterization of YM-90709 as a novel antagonist which inhibits the binding of interleukin-5 to interleukin-5 receptor. International Immunopharmacology, 2, 1693-1702. doi:10.1016/S1567-5769(02)00191-1

[19] O’Byrne, P.M. (2011) Therapeutic strategies to reduce asthma exacerbations. Journal of Allergy and Clinical Immunology, 128, 257-263. doi:10.1016/j.jaci.2011.03.035

[20] Busse, W.W., Ring, J., Huss-Marp, J. and Kahn, J.E. (2010) A review of treatment with mepolizumab, an antiIL-5 mAb, in hypereosinophilic syndromes and asthma. Journal of Allergy and Clinical Immunology, 125, 803813. doi:10.1016/j.jaci.2009.11.048

[21] Wenzel, S.E., Schwartz, L.B., Langmack, E.L., Halliday, J.L., Trudeau, J.B., Gibbs, R.L. and Chu, H.W. (1999) Evidence that severe asthma can be divided pathologically into two inflammatory subtypes with distinct physiologic and clinical characteristics. American Journal of Respiratory and Critical Care Medicine, 160, 1001-1008.

[22] Sexton, D.W., Blaylock, M.G. and Walsh, G. (2001) Human alveolar epithelial cells engulf apoptotic eosinophils by means of integrin- and phosphatidylserine receptordependent mechanisms: A process upregulated by dexamethasone. Journal of Allergy and Clinical Immunology, 108, 962-969.

[23] Schleimer, R.P. and Bochner, B.S. (1994) The effects of glucocorticoids on human eosinophils. Journal of Allergy and Clinical Immunology, 94, 1202-1213. doi:10.1016/0091-6749(94)90333-6

[24] Adachi, T., Motojima, S., Hirata, A., Fukuda, T., Kihara, N. and Kosaku, H. (1996) Eosinophil apoptosis caused by theophylline, glucocorticoids, and macrolides after stimulation with IL-5. Journal of Allergy and Clinical Immunology, 98, S207-S215. doi:10.1016/S0091-6749(96)70068-4

[25] Sexton, D.W., Al-Rabia, M.W., Blaylock, M.G. and Walsh, G.M. (2004) Phagocytosis of apoptotic eosinophils but not neutrophils by bronchial epithelial cells. Clinical \& Experimental Allergy, 34, 1514-1524. doi:10.1111/j.1365-2222.2004.02054.X

[26] Schleimer, R.P. (1990) Effects of glucocorticoids on inflammatory cells relevant to their therapeutic applications in asthma. American Review of Respiratory Disease, 141, S59-S69.

[27] Zhang, X., Moilanen, E. and Kankaanranta, H. (2000) Enhancement of human eosinophil apoptosis by fluticasone propionate, budesonide, and beclomethasone. European Journal of Pharmacology, 496, 325-332. doi:10.1016/S0014-2999(00)00690-7

[28] Meagher, L.C., Cousin, J.M., Seckl, J.R. and Haslett, C. (1996) Opposing effects of glucocorticoids on the rate of apoptosis in neutrophilic and eosinophilic granulocytes. The Journal of Immunology, 156, 4422-4428.

[29] Chauhan, S., Leach, C.H., Kunz, S., bloom, J.W. and Miesfeld, R.L. (2003) Glucocorticoid regulation of human eosinophil gene expression. Journal of Steroid Bio- 
chemistry and Molecular Biology, 84, 441-452. doi:10.1016/S0960-0760(03)00065-7

[30] Lilly, C.M., Nakamura, H., Kesselman, H., NaglerAnderson, C., Asano, K. and Garcia, Z.E.A. (1997) Expression of eotaxin by human lung epithelial cells-induction by cytokines and inhibition by glucocorticoids. Journal of Clinical Investigation, 99, 1767-1773. doi:10.1172/JCI119341

[31] Ren, Y. and Savill, J. (1995) Proinflammatory cytokines potentiate thrombospondin-mediated phagocytosis of neutrophils undergoing apoptosis. The Journal of Immunology, 154, 2366-2374.

[32] Saunders, M.W., Wheatley, A.H., George, S.J., Lai, T. and Birchall, M.A. (1999) Do corticosteroids induce apoptosis in nasal polyp inflammatory cells? In Vivo and in Vitro studies. Laryngoscope, 109, 785-790. doi:10.1097/00005537-199905000-00019

[33] Woolley, K.L., Gibson, P.G., Carty, K., Wilson, A.J., Twaddell, S.H. and Woolley, M.J. (1996) Eosinophil apoptosis and the resolution of airway inflammation in asthma. American Journal of Respiratory and Critical Care Medicine, 154, 237-243.

[34] Liu, Y.Q., Cousin, J.M., Hughes, J., Van Damme, J., Seckl, J.R. and Haslett, C. (1999) Glucocorticoids promote nonphlogistic phagocytosis of apoptotic leukocytes. The Journal of Immunology, 162, 3639-3646.

[35] Duval, E., Wyllie, A.H. and Morris, R.G. (1985) Macrophage recognition of cells undergoing programmed cell death (apoptosis). Immunology, 56, 351-358.

[36] Savill, J., Hogg, N., Ren, Y. and Haslett, C. (1992) Thrombospondin co-operates with CD36 and the vitronectin receptor in macrophage recognition of neutrophils undergoing apoptosis. Journal of Clinical Investigation, 90, 1513-1522. doi:10.1172/JCI116019

[37] Albert, M.L., Pearce, S.F.A., Francisco, L.M., Sauter, B., Roy, P. and Silverstein, R.L. (1998) Immature dendritic cells phagocytose apoptotic cells via $\alpha \mathrm{v} \beta 5$ and CD36 and cross present antigens to cytotoxic $\mathrm{T}$ lymphocytes. The Journal of Experimental Medicine, 188, 1359-1368. doi:10.1084/jem.188.7.1359

[38] Finnemann, S.C., Bonilha, V.L., Marmorstein, A.D. and Rodriguez-Boulan, E. (1997) Phagocytosis of rod outer segments by retinal pigmented epithelial cells requires $\alpha \mathrm{v} \beta 5$ integrin for binding but not internalization. Proceedings of the National Academy of Sciences of the United States of America, 94, 12932. doi:10.1073/pnas.94.24.12932

[39] Fadok, V.A., Savill, J.S., Haslett, C., Bratton, D.L., Doherty, D.E. and Campbell, PA. (1992) Different populations of macrophages use either the vitronectin receptor or the phosphatidylserine receptor to recognize and remove apoptotic cells. The Journal of Immunology, 149, 4029-4035.
[40] Fadok, V.A., Voelker, D.R., Campbell, P.A., Cohen, J.J., Bratton, D.L. and Henson, P.M. (1992) Exposure of phosphatidyl-serine on the surface of apoptotic lymphocytes triggers specific recognition and removal by macrophages. The Journal of Immunology, 148, 2207-2216.

[41] Platt, N., Suzuli, H., Kurihara, Y., Kodoma, T. and Gordon, S. (1996) Role for the class A scavenger receptor in the phagocytosis of apoptotic thymocytes in vitro. Proceedings of the National Academy of Sciences of the United States of America, 93, 12456-12460. doi:10.1073/pnas.93.22.12456

[42] Sambrano, G.R. and Steinberg, D. (1995) Recognition of oxidatively damaged and apoptotic cells by an oxidized low-density lipoprotein receptor on mouse peritoneal macrophages: Role of membrane phosphatidylserine. Proceedings of the National Academy of Sciences of the United States of America, 92, 1396-1400. doi:10.1073/pnas.92.5.1396

[43] Walsh, G., Sexton, D.W., Blaylock, M.G. and Convery, C.M. (1999) Resting and cytokine-stimulated human small airway epithelial cells recognize and ingest apoptotic eosinophils. Blood, 94, 2827-2835.

[44] Walsh, G.M. (1999) Advances in the immunobiology of eosinophils and their role in disease. Critical Reviews in Clinical Laboratory Sciences, 36, 453-496. doi:10.1080/10408369991239277

[45] Fadok, V.A., Bratton, D.L., Rose, D.M., Pearson, A., Ezekewitz, R.A.B. and Henson, P.M. (2000) A receptor for phosphatidylserine-specific clearance of apoptotic cells. Nature, 405, 85-90. doi:10.1038/35011084

[46] Fadok, V., Bratton, D.L., Frasch, S.C., Warner, M. and Henson, P.M. (1998) The role of phosphatidylserine in recognition of apoptotic cells by phagocytes. Cell Death \& Differentiation, 5, 551-562. doi:10.1038/sj.cdd.4400404

[47] Fadok, V.A., de Cathelineau, A., Daleke, D.L., Henson, P.M. and Bratton, D.L. (2001) Loss of phospholipid asymmetry and surface exposure of phosphatidylserine is required for phagocytosis of apoptotic cells by macrophages and fibroblasts. The Journal of Biological Chemistry, 276, 1071-1077. doi:10.1074/jbc.M003649200

[48] Alenzi, F.Q. (2009) Role of apoptosis in airway epithelium. Pakistan Journal of Physiology, 5, 1-10.

[49] Alenzi, F.Q. (2008) Apoptosis and eosinophils. Regulation and clinical relevance. Saudi Medical Journal, 29, 643-656.

[50] Alenzi, F.Q., Alenazi, B., Alanzy, F., Mubaraki, A., Salem, M., Al-Jabri, A., Lotfy, M., Bamaga, M., AlRabia, M. and Richard, K.H. (2010) The role of caspase activation and mitochondrial depolarisation in cultured human apoptotic eosinophils. Saudi Journal of Biological Sciences, 17, 29-36. 